\title{
Green Synthesis of Symmetric Dimaleamic Acids from Dianilines and Maleic Anhydride: Behind New Bidentate Ligands for MOFs ${ }^{\dagger}$
}

\author{
Julio C. Flores-Reyes ${ }^{1}$, José L. Sosa-Juárez ${ }^{1}$, Mayra Sánchez-Serratos ${ }^{1}$, Perla Islas-Jácome ${ }^{1}$, \\ Atilano Gutiérrez-Carrillo ${ }^{1}$, Francisco Méndez ${ }^{1,2,3}$, Galdina V. Suárez-Moreno ${ }^{4, *}$, Alejandro Islas-Jácome 1,* and \\ Eduardo González-Zamora 1,*
}

Citation: Flores-Reyes, J.C.; SosaJuárez, J.L.; Sánchez-Serratos, M.; Islas-Jácome, P.; Gutiérrez-Carrillo, A.; Méndez, F.; Suárez-Moreno, G.V.; Islas-Jácome, A.; GonzálezZamora, E. Green Synthesis of Symmetric Dimaleamic Acids from Dianilines and Maleic Anhydride: Behind New Bidentate Ligands for MOFs. Chem. Proc. 2021, 3, 92. https://doi.org/10.3390/ecsoc-2408379

Academic Editors: Julio A. Seijas and M. Pilar Vázquez-Tato

Published: 14 November 2020

Publisher's Note: MDPI stays neutral with regard to jurisdictional claims in published maps and institutional affiliations.

Copyright: $(\odot 2020$ by the authors. Licensee MDPI, Basel, Switzerland. This article is an open access article distributed under the terms and conditions of the Creative Commons Attribution (CC BY) license (http://creativecommons.org/licenses/by/4.0/).
1 Departamento de Química, Universidad Autónoma Metropolitana-Iztapalapa, San Rafael Atlixco 186, Col. Vicentina, Iztapalapa, Ciudad de México C.P. 09340, Mexico; flores.reyes.jc@gmail.com (J.C.F.-R.); sosajuarz@gmail.com (J.L.S.-J.); mayra.schz@gmail.com (M.S.-S.); permau26@gmail.com (P.I.-J.); agrmn@xanum.uam.mx (A.G.-C.); fm@xanum.uam.mx (F.M.)

2 Le Studium, Loire Valley Institute for Advanced Studies, 4500 Orleans \& Tours, France;

3 Haute Température et Irradiation (CEMHTI), UPR3079 CNRS, CEMHTI 1, Avenue de la Recherche Scientifique, 45071 Orleans, France

4 Instituto Politécnico Nacional, Unidad Profesional Interdisciplinaria de Biotecnología, Av. Acueducto S/N, Barrio la Laguna Ticomán, CDMX C.P. 07340, Mexico

* Correspondence: gsuarezm@ipn.mx (G.V.S.-M.); aij@xanum.uam.mx (A.I.-J.); egz@xanum.uam.mx (E.G.-Z.)

+ Presented at the 24th International Electronic Conference on Synthetic Organic Chemistry, 15 November-15 December 2020; Available online: https://sciforum.net/conference/ecsoc-24.

\begin{abstract}
We herein report the synthesis and characterization of six $\alpha, \beta$-unsaturated dicarboxylic acid ligands with different phenyl spacers, and two ligands with a biphenyl and anthraquinone spacers. All these dimaleamic acids were synthesized in 16 to $99 \%$ yields via a base-catalyzed maleimide ring opening in water (ligand 2), or by a di- $N$-acylation from the corresponding diamines and maleic anhydride in acetic acid (ligands 4, 6, 8, 10, 12, 14 and 16). These reactions were performed using green solvents, while requiring minimal work up procedures, making them suitable alternatives to access these types of bidentate ligands quickly, which can be used to fabricate new metal-organic frameworks (MOFs).
\end{abstract}

Keywords: metal-organic frameworks (MOFs); bidentate ligands; dicarboxylates; maleic anhydride; dimaleimides; dimaleamic acids; green chemistry

\section{Introduction}

Metal-organic frameworks (MOFs) are hybrid structures composed by the union, via coordination bonds, of metal ions or metal clusters to organic ligands with donor atoms, producing crystalline and often highly porous materials through a repetitive network that propagates into one, two or three dimensions [1]. Given that the amount of ligands and metal salts that can be combined for MOF synthesis is practically unlimited, MOFs can be tailored according to their intended use, and have therefore been used in several applications of economic, technological and environmental importance such as luminescence and sensing [2], electrochemistry [3], catalysis [4], gas capture, storage and separation [5] and biomedicine [6]. Most of the ligands used to construct MOFs are neutral or anionic. The drawback of using neutral ligands is the requirement of counter ions due to the positive charge generated by the formation of the coordination bond, while anionic ligands do not have this disadvantage because they bind metal atoms via a charge compensation [7]. Pyridine and pyrazine are among the most common donor groups present on neutral lig- 
ands, while for anionic ligands, carboxylates are the most common ones [8]. The carboxylate group can bind to a metal atom in a monodentate or bidentate manner, the latter of which produces the strongest binding. When bidentate binding occurs, it causes the insitu formation of inorganic clusters called "secondary building units" (SBUs), which confer great rigidity to the framework being constructed and facilitate its self-assembly [9]. The spacers used in the ligand's backbone also play a key role in MOF synthesis. As a result, most ligands have bound or fused aromatic rings as central or extending units because their rigidity can benefit the crystal packing and arrangement, for instance, via $\pi-\pi$ stacking-type interactions [10].

Maleamic acid is a nitrogen-containing analog of maleic acid. $\mathrm{N}$-substituted maleamic acids are highly conjugated, and their use as polydentate ligands has been well documented with lanthanide ions such as $\mathrm{La}^{3+}[11], \mathrm{Eu}^{3+}, \mathrm{Tb}^{3+}$ and $\mathrm{Yb}^{3+}[12]$, and transition metals such as $\mathrm{Cu}^{2+}[13,14]$. The most common method for maleamic acid synthesis involves the reaction between a primary amine and maleic anhydride under mild reaction conditions (Scheme 1), in a wide variety of solvents, for instance, dichloromethane [15], diethyl ether [16], toluene [17], tetrahydrofuran [18] or xylene [19], which may pose health and environmental hazards.

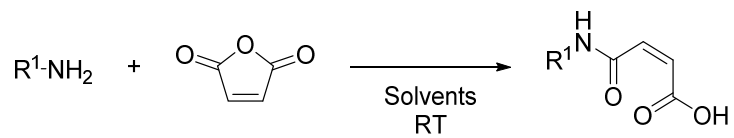

Scheme 1. General reaction scheme for the synthesis of maleamic acids.

The main objective of this work is to provide environmentally benign procedures for the synthesis of ligands that can be used to construct novel MOF structures. To do this, we present the synthesis and spectroscopic characterization of eight bis-maleamic acid ligands, the first of which (ligand 2) was synthesized in water as the solvent from a bis-maleimide, and the rest in acetic acid from the corresponding diamines and maleic anhydride. Also, ligands 12 and 16 have not been previously reported, and their structures show that this procedure can be applicable to fabricate ligands with a more complex backbone.

\section{Results and Discussion}

\subsection{Synthesis of (2E,2'E)-4,4'-(1,4-phenylenebis(azanediyl))bis(4-oxobut-2-enoic acid) (2)}

For the synthesis of the ligand $\mathbf{2}$, the $N, N^{\prime}$-(1,4-phenylene)dimaleimide 1 was reacted in an aqueous solution $[0.1 \mathrm{M}]$ of sodium hydroxide for $3 \mathrm{~h}$ to afford the target molecule $\left(2 E, 2^{\prime} E\right)-4,4^{\prime}-(1,4$-phenylenebis(azanediyl))bis(4-oxobut-2-enoic acid) in an almost quantitative yield $(99 \%)$ via a base-catalyzed maleimide ring opening reaction (Scheme 2$)$. This methodology has been utilized to obtain maleamic acids [20-22], but, to the best of our knowledge, the hydrolysis of a bis-maleimide such as $\mathbf{1}$ to produce a bis-maleamic acid has not been reported yet.

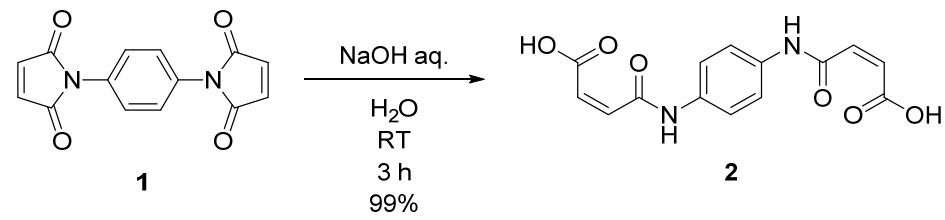

Scheme 2. Synthesis of the ligand 2.

The bis-carboxylic acid 2 was characterized by its physicochemical properties, as well as by spectroscopic techniques. Figure 1 shows the ${ }^{1} \mathrm{H}$ spectrum of compound $\mathbf{2}$. There is a broad singlet at $13.24 \mathrm{ppm}$, which is characteristic of the acidic protons from the carboxylic acid moiety, as well as a key singlet at $10.46 \mathrm{ppm}$, which corresponds to $\mathrm{NH}$ (amide protons). There is also a singlet at $7.60 \mathrm{ppm}$ corresponding to the four $\mathrm{H}$ atoms from the 
aromatic ring, and a couple of doublets at 6.47 and $6.31 \mathrm{ppm}$, respectively, which correspond to the alkene moiety.

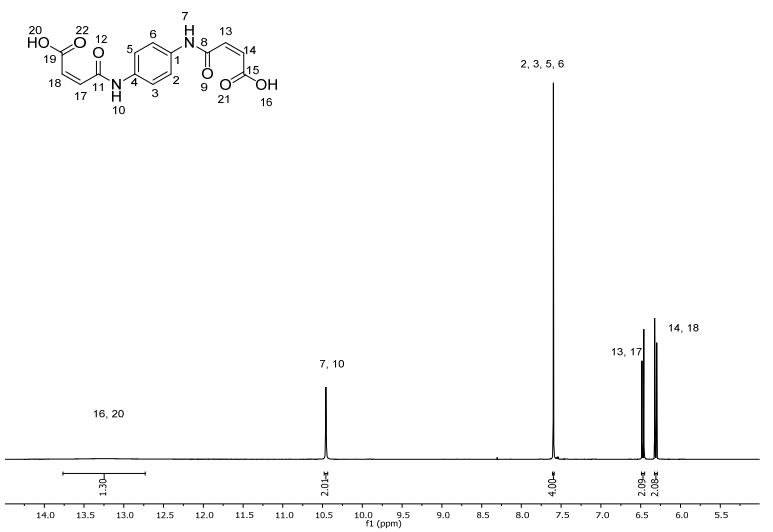

Figure 1. ${ }^{1} \mathrm{H}$ NMR spectrum of compound 2.

2.2. Synthesis of $\left(2 Z, 2^{\prime} Z\right)-4,4^{\prime}-(($ methylenebis(4,1-phenylene $))$ bis(azanediyl))bis(4-oxobut-2enoic acid) (4)

4,4'-methylenedianiline (3) was reacted with a slight excess of maleic anhydride for $1 \mathrm{~h}$ in acetic acid as solvent to generate the desired compound $\left(2 \mathrm{Z}, 2^{\prime} \mathrm{Z}\right)-4,4^{\prime}-(($ methylenebis(4,1-phenylene))bis(azanediyl))bis(4-oxobut-2-enoic acid) (4) in $62 \%$ yield (Scheme 3).

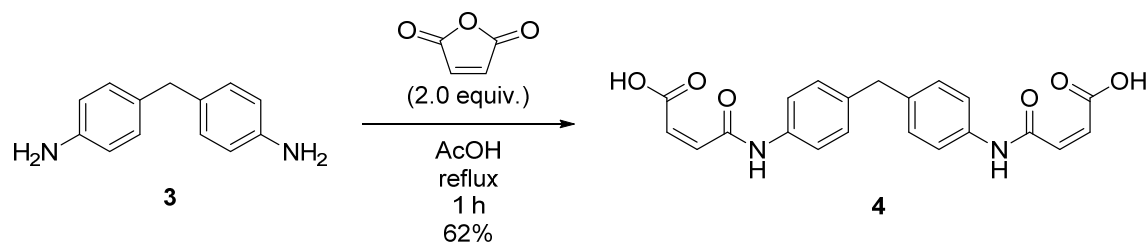

Scheme 3. Synthesis of ligand 4.

Compound 4 was characterized by its spectroscopic properties. Figure 2a shows the ${ }^{1} \mathrm{H}$ NMR spectra of compound 4 . The broad singlet corresponding to the carboxylic acid protons can be found at $13.07 \mathrm{ppm}$, followed by the singlet attributed to the $\mathrm{NH}$ protons at $10.43 \mathrm{ppm}$. Next, there are two doublets at 7.54 and $7.17 \mathrm{ppm}$ corresponding to the $\mathrm{A}^{2} \mathrm{~B}^{2}$ system of the aromatic rings, and finally another set of two doublets that correspond to the alkene protons. Figure $2 \mathrm{~b}$ shows the ${ }^{13} \mathrm{C}$ NMR spectrum. There are two key peaks at 166.7 and $163.1 \mathrm{ppm}$, respectively, which correspond to both carbonyl carbons, as well as another peak at $40 \mathrm{ppm}$ belonging to the methylene carbon.

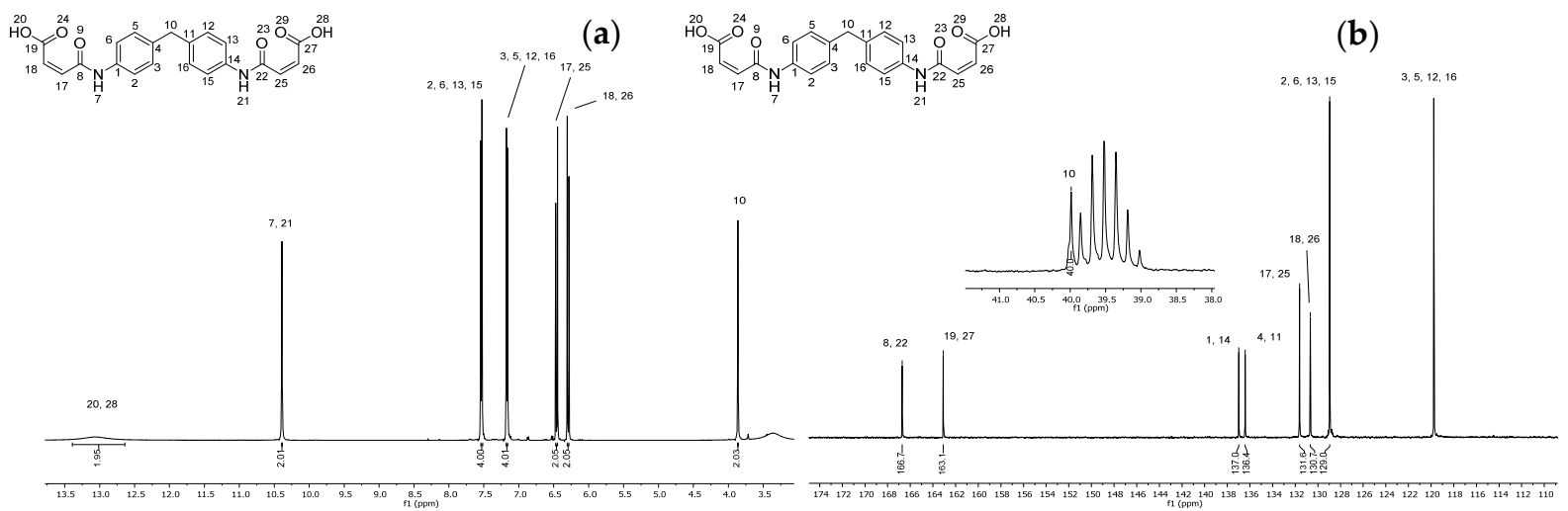

Figure 2. (a) ${ }^{1} \mathrm{H}$ NMR spectrum of ligand 4. (b) ${ }^{13} \mathrm{C}$ NMR spectrum of ligand 4. 
2.3. Synthesis of (2Z,2'Z)-4,4'-((oxybis(4,1-phenylene))bis(azanediyl))bis(4-oxobut-2-enoic acid) (6)

4,4'-oxydianiline (5) was reacted with two equivalents of maleic anhydride for $1 \mathrm{~h}$ in acetic acid as solvent to generate the desired compound (2Z,2'Z)-4, $4^{\prime}-(($ oxybis $(4,1-$ phenylene))bis(azanediyl))bis(4-oxobut-2-enoic acid) (6) via an $\mathrm{N}$-acylation reaction, in 50\% yield (Scheme 4).

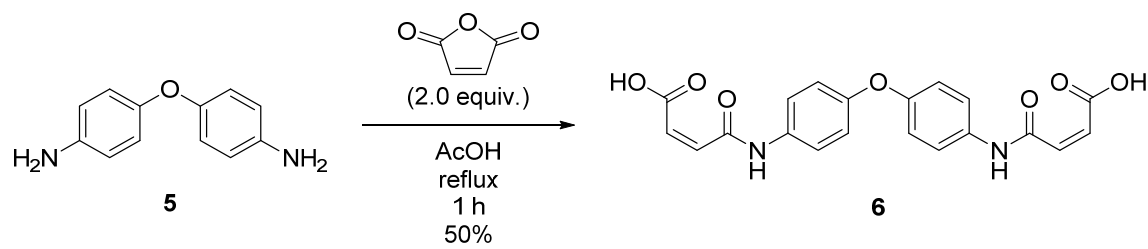

Scheme 4. Synthesis of ligand 6.

The ligand 6 was characterized by its spectroscopic properties. Figure 3a shows the ${ }^{1} \mathrm{H}$ NMR spectra of compound 6 . There is a key broad singlet corresponding to the carboxylic acid protons at $13.12 \mathrm{ppm}$ followed by the singlet attributed to the $\mathrm{NH}$ protons at $10.43 \mathrm{ppm}$, the set of two doublets at 7.62 and $6.98 \mathrm{ppm}$ corresponding to the $\mathrm{A}^{2} \mathrm{~B}^{2}$ system of the aromatic rings and finally another set of two doublets corresponding to the vinylic protons. The ${ }^{13} \mathrm{C}$ NMR spectrum shows the expected eight signals, with the key peaks at 166.6 and $163.0 \mathrm{ppm}$ corresponding to both pairs of carbonyl carbons (Figure $3 \mathrm{~b}$ ).

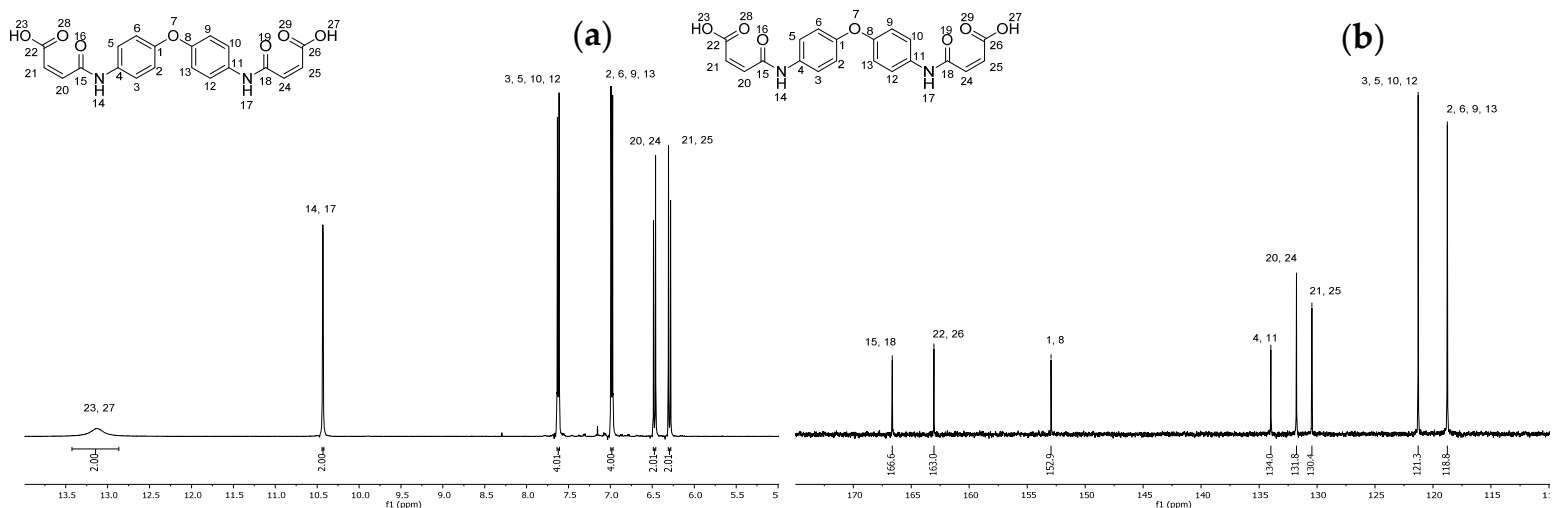

Figure 3. (a) ${ }^{1} \mathrm{H}$ NMR spectrum of ligand 6. (b) ${ }^{13} \mathrm{C}$ NMR spectrum of ligand 6.

\subsection{Synthesis of (2Z,2'Z)-4,4'-((thiobis(4,1-phenylene))bis(azanediyl))bis(4-oxobut-2-enoic acid)} (8)

4,4'-thiodianiline (7) reacted with two equivalents of maleic anhydride for $1 \mathrm{~h}$ in acetic acid as the solvent furnishing the desired compound (2Z,2'Z)-4,4'-((thiobis(4,1-phenylene))bis(azanediyl))bis(4-oxobut-2-enoic acid) (8) via an $\mathrm{N}$-acylation reaction, in 55\% yield (Scheme 5).

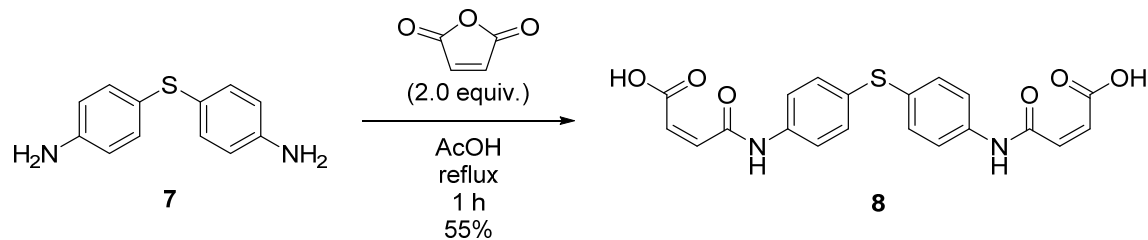

Scheme 5. Synthesis of ligand 8. 
Ligand 8 was characterized by spectroscopic techniques. Figure 4a shows the ${ }^{1} \mathrm{H}$ NMR spectrum, which shows the key broad singlet at $12.97 \mathrm{ppm}$ that belongs to the carboxylic acid protons and another singlet for the $\mathrm{NH}$ protons at $10.47 \mathrm{ppm}$. There are two sets of two doublets, the first at 7.63 and $7.29 \mathrm{ppm}$ corresponding to the $\mathrm{A}^{2} \mathrm{~B}^{2}$ system of the aromatic rings, and the following set belonging to the alkene protons. Figure $4 \mathrm{~b}$ shows the ${ }^{13} \mathrm{C}$ NMR spectrum, which shows the expected eight signals, the two key peaks being at 166.8 and $163.3 \mathrm{ppm}$ and corresponding to the carbonyl carbons.

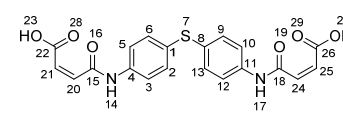

(a)

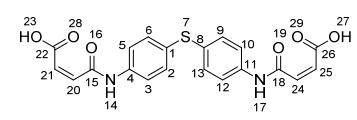

(b)

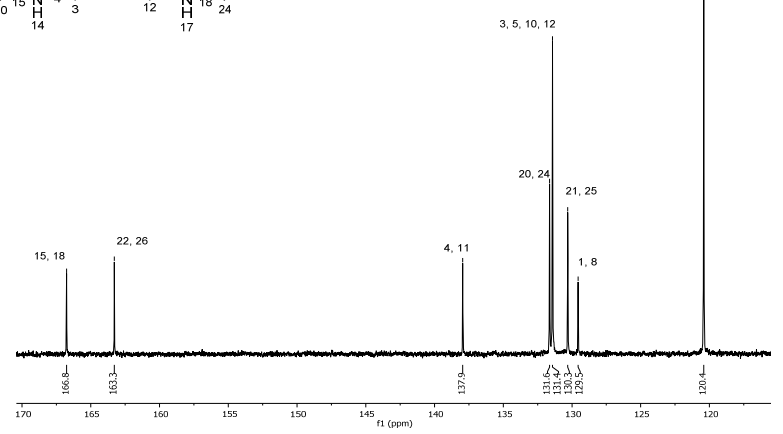

Figure 4. (a) ${ }^{1} \mathrm{H}$ NMR spectrum of ligand 6. (b) ${ }^{13} \mathrm{C}$ NMR spectrum of ligand 8.

2.5. Synthesis of (2Z,2'Z)-4,4'-((sulfonylbis(4,1-phenylene))bis(azanediyl))bis(4-oxobut-2-enoic acid) (10)

The 4,4'-sulfonyldianiline (9) reacted with two equivalents of maleic anhydride for 1 $\mathrm{h}$ in acetic acid as the solvent furnishing the target compound $\left(2 \mathrm{Z}, 2^{\prime} Z\right)-4,4^{\prime}-((\mathrm{sul}-$ fonylbis(4,1-phenylene))bis(azanediyl))bis(4-oxobut-2-enoic acid) (10) via an $N$-acylation reaction, in 39\% yield (Scheme 6).

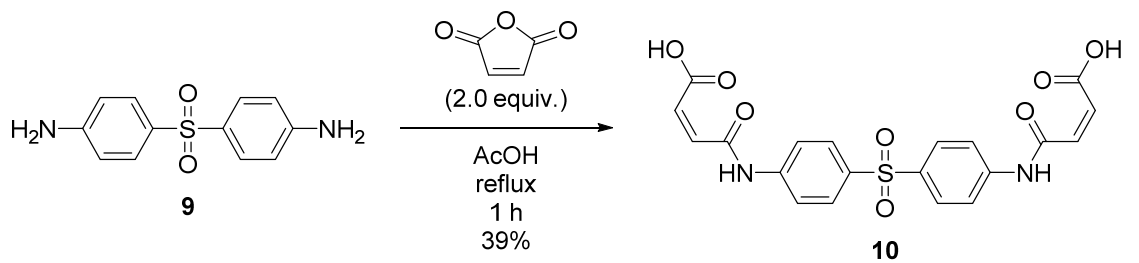

Scheme 6. Synthesis of compound 10.

Thus, ligand 10 was characterized by spectroscopic techniques. Figure 5 a shows the ${ }^{1} \mathrm{H}$ NMR spectrum, which shows the key broad singlet at $12.86 \mathrm{ppm}$ that corresponds to the carboxylic acid protons and another singlet for the $\mathrm{NH}$ protons at $10.68 \mathrm{ppm}$, and two sets of two doublets: the first at 7.88 and $7.82 \mathrm{ppm}$ corresponding to the $\mathrm{A}^{2} \mathrm{~B}^{2}$ system of the aromatic rings, and the following set belonging to the alkene protons. Figure $5 \mathrm{~b}$ shows the ${ }^{13} \mathrm{C}$ NMR spectrum, which shows the expected eight signals, the two key peaks being at 166.8 and $163.8 \mathrm{ppm}$, and corresponding to the carbonyl carbons. 


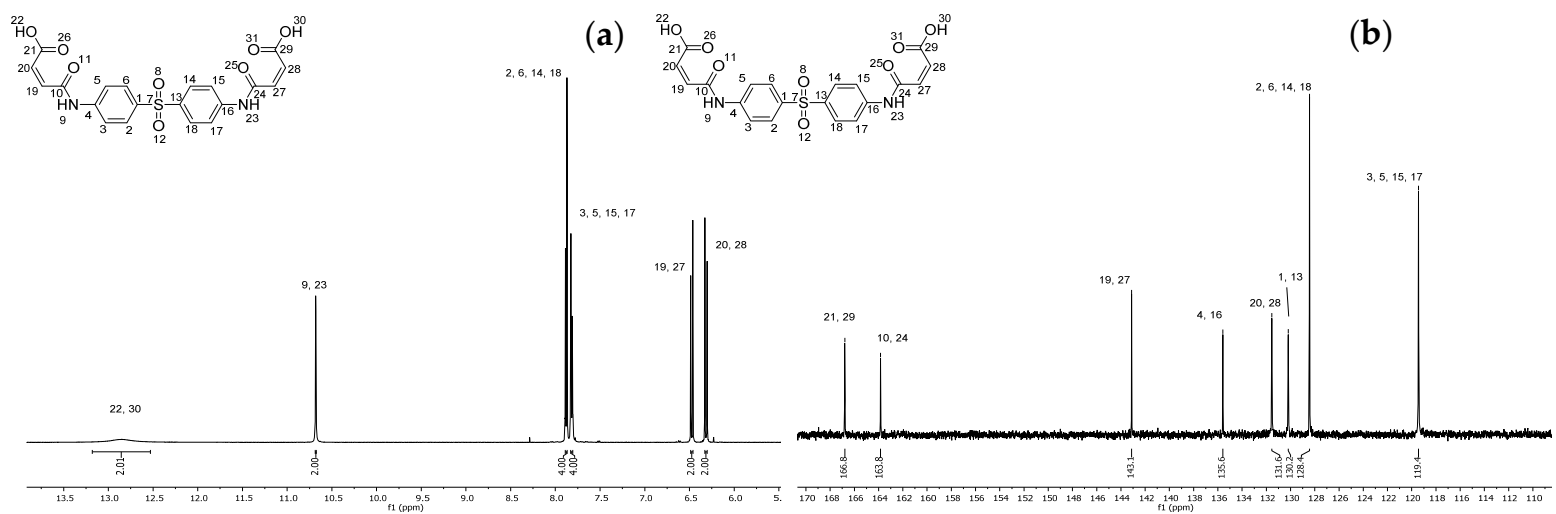

Figure 5. (a) ${ }^{1} \mathrm{H}$ NMR spectrum of ligand 10. (b) ${ }^{13} \mathrm{C}$ NMR spectrum of ligand 10.

2.6. Synthesis of $\left(2 Z, 2^{\prime} Z\right)-4,4^{\prime}-((9,10-$ dioxo-9,10-dihydroanthracene-2,6diyl)bis(azanediyl))bis(4-oxobut-2-enoic acid) (12)

2,6-diaminoanthracene-9,10-dione (11) reacted with two equivalents of maleic anhydride for $1 \mathrm{~h}$ in acetic acid as the solvent furnishing the target compound $\left(2 \mathrm{Z}, 2^{\prime} \mathrm{Z}\right)-4,4^{\prime}$ ((9,10-dioxo-9,10-dihydroanthracene-2,6-diyl)bis(azanediyl))bis(4-oxobut-2-enoic acid) (12) via an $N$-acylation reaction, in $22 \%$ yield (Scheme 7 ).

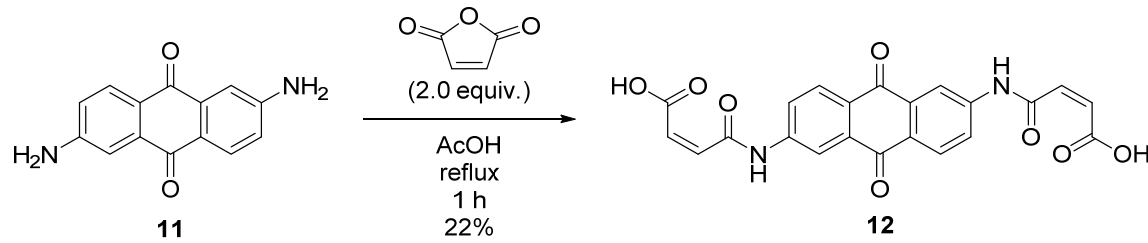

Scheme 7. Synthesis of ligand 12.

Ligand 12 was characterized by spectroscopic techniques. Figure 6 a shows the ${ }^{1} \mathrm{H}$ NMR spectrum, which shows the key broad singlet at $12.88 \mathrm{ppm}$ that corresponds to the carboxylic acid protons, and another singlet for the NH protons at $10.93 \mathrm{ppm}$. Three doublets at 8.47, 8.17 and 8.07 ppm correspond to the trisubstituted phenyl rings and two doublets of the alkene protons. Figure $6 \mathrm{~b}$ shows the ${ }^{13} \mathrm{C}$ NMR spectrum, which shows the expected 11 signals, the two key peaks being at 166.9 and $164.0 \mathrm{ppm}$, corresponding to the carbonyl from the amide and carboxylic acid, and another peak at $181.2 \mathrm{ppm}$, which accounts for both carbonyls of the anthraquinone core.

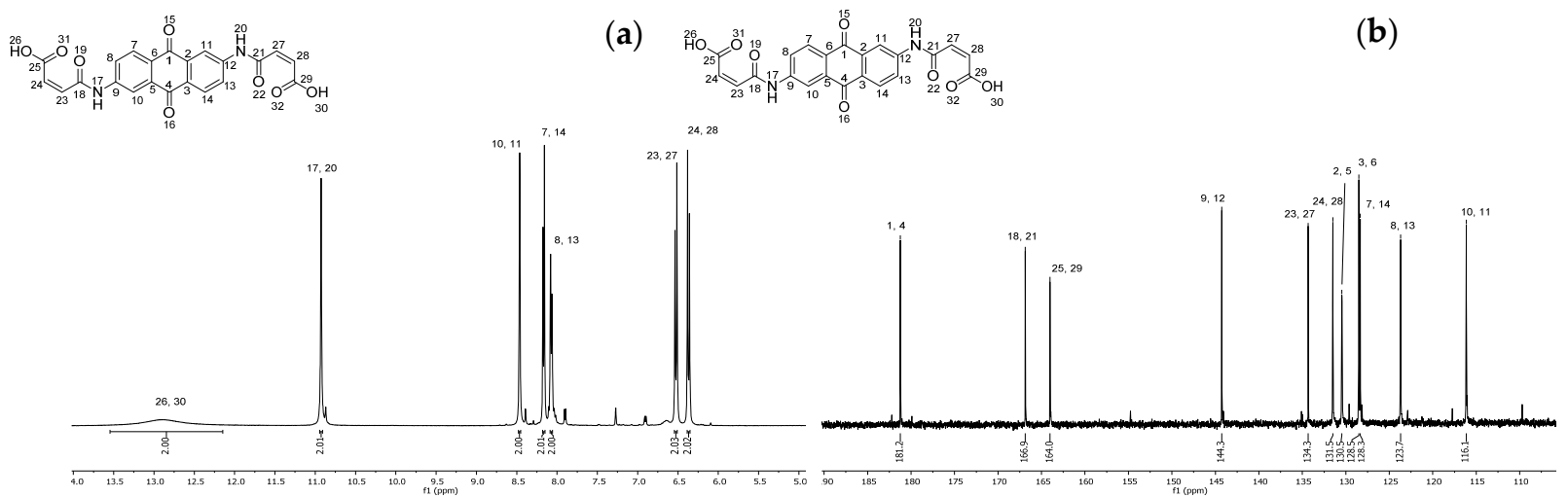

Figure 6. (a) ${ }^{1} \mathrm{H}$ NMR spectrum of ligand 12. (b) ${ }^{13} \mathrm{C}$ NMR spectrum of ligand 12. 
2.7. Synthesis of (2Z,2'Z)-4,4'-((disulfanediylbis(4,1-phenylene))bis(azanediyl))bis(4-oxobut-2enoic acid) (14)

For the synthesis of ligand 14, 4,4'-disulfanediyldianiline (13) was reacted with two equivalents of maleic anhydride for $1 \mathrm{~h}$ in acetic acid as the solvent furnishing the target compound (2Z,2'Z)-4,4'-((disulfanediylbis(4,1-phenylene))bis(azanediyl))bis(4-oxobut-2enoic acid) (14) via an $N$-acylation reaction, in $34 \%$ yield (Scheme 8 ).
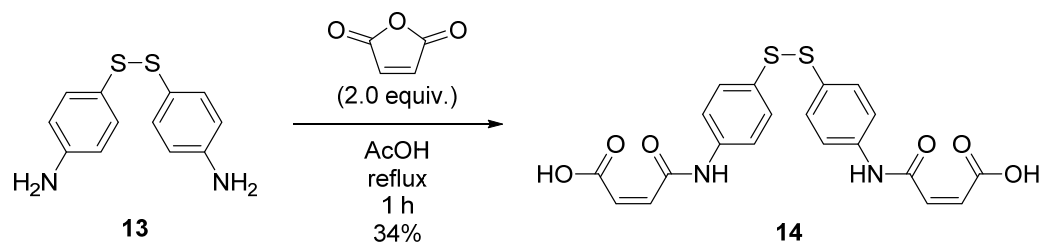

Scheme 8. Synthesis of ligand disulfane bridged 14.

Ligand 14 was characterized by spectroscopic techniques. Figure 7 a shows the ${ }^{1} \mathrm{H}$ NMR spectrum, which shows the key broad singlet at $12.94 \mathrm{ppm}$ that corresponds to the carboxylic acid protons and another singlet for the NH protons at $10.50 \mathrm{ppm}$. The two doublets corresponding to the $\mathrm{A}^{2} \mathrm{~B}^{2}$ system of the phenyl rings are present at 7.64 and 7.47 $\mathrm{ppm}$, followed by the two doublets of the alkene protons at 6.46 and $6.30 \mathrm{ppm}$. Figure $7 \mathrm{~b}$ shows the ${ }^{13} \mathrm{C}$ NMR spectrum, which shows the expected seven signals, the two key peaks being at 166.8 and $163.3 \mathrm{ppm}$, and corresponding to the carbonyl carbons.

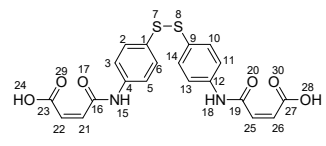

(a)

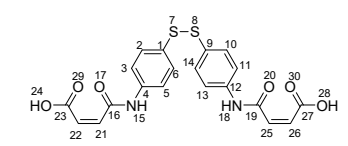

(b)

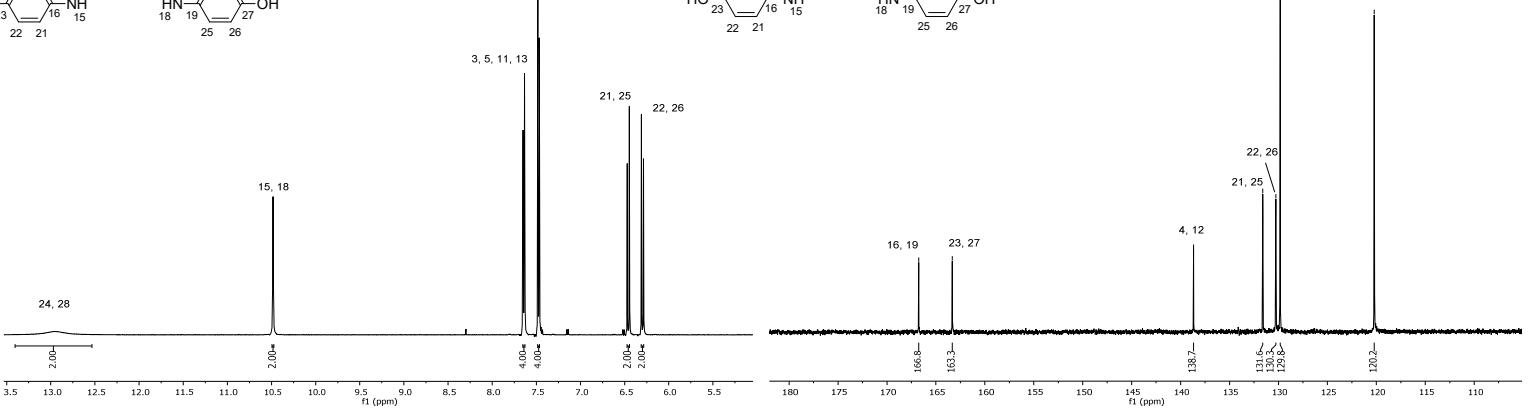

Figure 7. (a) ${ }^{1} \mathrm{H}$ NMR spectrum of disulfane bridged ligand 14. (b) ${ }^{13} \mathrm{C}$ NMR spectrum of disulfane bridged ligand 14.

\subsection{Synthesis of $\left(2 Z, 2^{\prime} Z\right)-4,4^{\prime}-\left(\left(\left(\left[1,1^{\prime}-\right.\right.\right.\right.$-biphenyl $]-4,4^{\prime}$-diylbis $($ oxy $\left.)\right) b i s(4,1$ -} phenylene))bis(azanediyl))bis(4-oxobut-2-enoic acid) (16)

For the synthesis of ligand 16, 4,4'-([1,1'-biphenyl]-4,4'-diylbis(oxy))dianiline (15) was reacted with two equivalents of maleic anhydride for $1 \mathrm{~h}$ in acetic acid as the solvent furnishing the target compound (2Z,2'Z)-4,4'-((([1,1'-biphenyl]-4,4'-diylbis(oxy))bis(4,1-phenylene))bis(azanediyl))bis(4-oxobut-2-enoic acid) via an $\mathrm{N}$-acylation reaction, in $16 \%$ yield (Scheme 9).

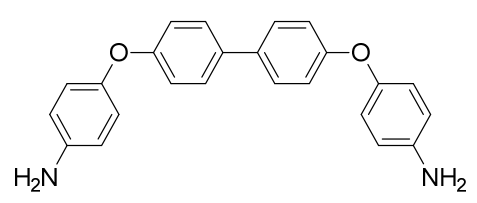

15
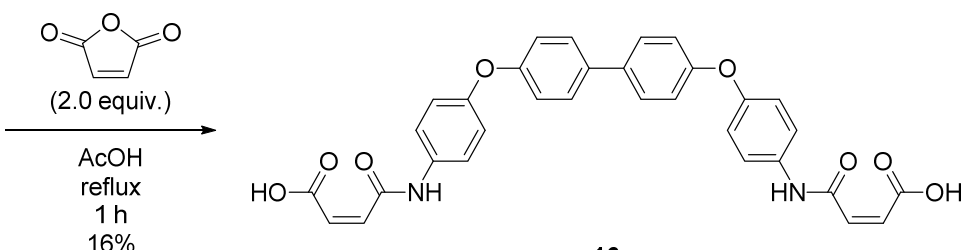

16

Scheme 9. Synthesis of ligand 16. 
Ligand 16 was characterized by spectroscopic techniques. Figure 8a shows the ${ }^{1} \mathrm{H}$ NMR spectrum, which shows the key broad singlet at $13.13 \mathrm{ppm}$ that corresponds to the carboxylic acid protons and another singlet for the NH protons at $10.46 \mathrm{ppm}$. In this case, there are two sets of two doublets that correspond to both non-equivalent (anisochrone) $\mathrm{A}^{2} \mathrm{~B}^{2}$ system of the four $p$-substituted phenyl rings at 7.66, 7.63, 7.06 and $7.05 \mathrm{ppm}$ followed by the two doublets of the alkene protons at 6.48 and $6.31 \mathrm{ppm}$. Figure $8 \mathrm{~b}$ shows the ${ }^{13} \mathrm{C}$ NMR spectrum, which shows 11 signals, the two key peaks being at 166.7 and $163.1 \mathrm{ppm}$, and corresponding to the carbonyl carbons.

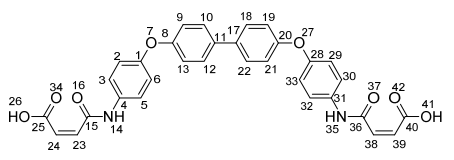

(a)

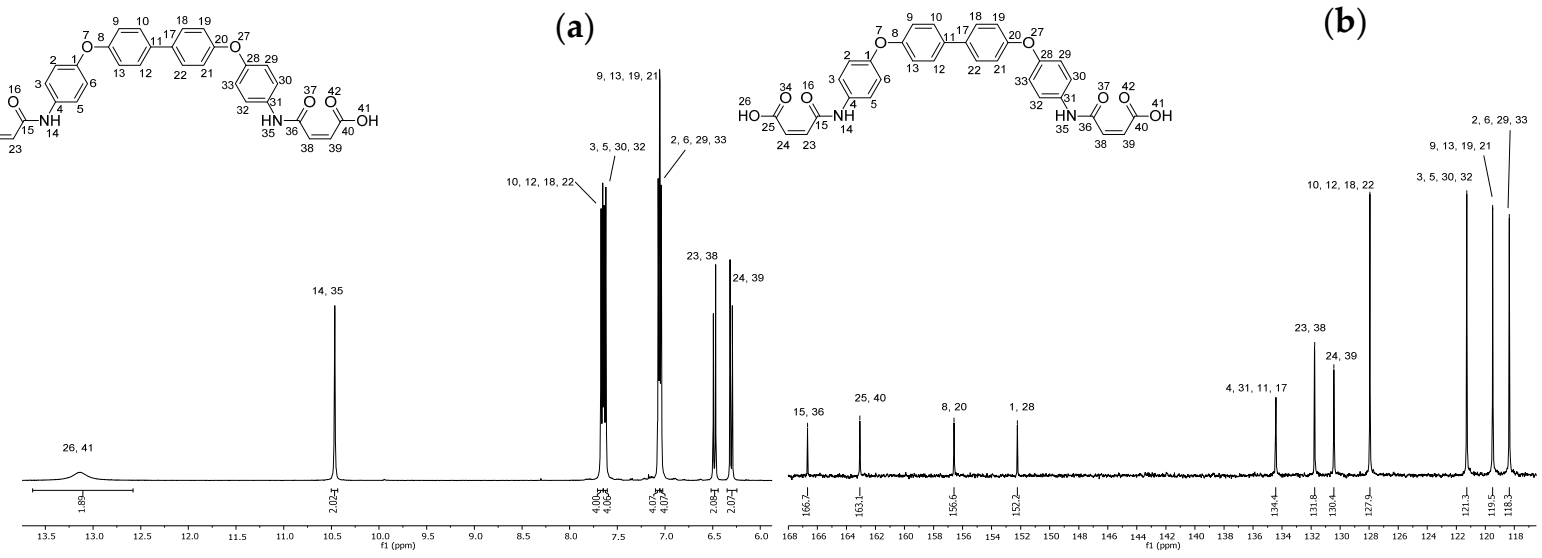

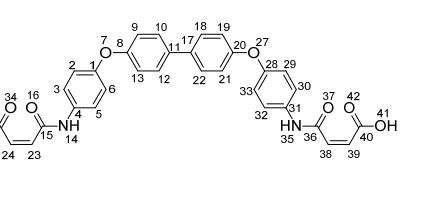

(b)

Figure 8. (a) ${ }^{1} \mathrm{H}$ NMR spectrum of ligand 16. (b) ${ }^{13} \mathrm{C}$ NMR spectrum of ligand 16.

\section{Conclusions}

The ligand 2 was synthesized via a base catalyzed bis-maleimide ring opening in water in near quantitative yield ( $>99 \%)$. Ligands $4, \mathbf{6}, \mathbf{8}, \mathbf{1 0}, \mathbf{1 2}, \mathbf{1 4}$ and 16 were synthesized via a reaction between the corresponding diamines and maleic anhydride in acetic acid as solvent in good to moderate yields (16-62\%). Furthermore, ligands 12 and 16 have not been previously reported. The described processes use green solvents, mild reaction conditions, and a minimal work-up procedure, which makes them attractive alternatives for the synthesis of ligands with potential application for fabricating new MOFs.

\section{Experimental Section}

\subsection{General Information, Instrumentation and Chemicals}

${ }^{1} \mathrm{H}$ and ${ }^{13} \mathrm{C}$ NMR spectra were acquired on a Bruker Advance III (500 MHz) spectrometer. The solvent was deuterated dimethyl sulfoxide ( $d^{6}$-DMSO). Chemical shifts are reported in parts per million (/ppm). The internal reference for NMR spectra is with respect to tetramethyl silane (TMS) at $0.0 \mathrm{ppm}$. Coupling constants are reported in Hertz (J/Hz). Multiplicities of the signals are reported using the standard abbreviations: singlet (s), doublet (d), triplet ( $\mathrm{t}$ ), quartet (q) and multiplet $(\mathrm{m})$. NMR data were treated using MestReNova software (12.0.0-20080). The reaction progress was monitored by thin layer chromatography (TLC) on precoated kieselgel 60 F254 plates, and the spots were visualized under UV light (254 or $365 \mathrm{~nm}$ ). Structural drawings were created using ChemDraw professional software (15.0.0.106). All starting materials were purchased from Sigma-Aldrich and were used without further purification or dehydration. The solvents were distilled and dried according to standard procedures.

\subsection{Synthesis of (2E,2'E)-4,4'-(1,4-phenylenebis(azanediyl))bis(4-oxobut-2-enoic acid) (2)}

In a $50 \mathrm{~mL}$ round-bottomed flask were added $0.27 \mathrm{~g}$ (1.0 equiv.) of 1,1'-(1,4-phenylene)bis(1H-pyrrole-2,5-dione) (1) into $20 \mathrm{~mL}$ of distilled water to obtain a turbid solution. Then, an aqueous solution of sodium hydroxide $(10 \mathrm{~mL}, 0.5 \mathrm{M})$ was poured slowly into the mixture, avoiding surpassing a $\mathrm{pH}$ of 12 at room temperature. After $3 \mathrm{~h}$ of stirring, a yellow solution was obtained. This was acidified with $[3 \mathrm{M}] \mathrm{HCl}$ until a $\mathrm{pH}$ of 2 was 
reached. This prompted a yellow precipitate to appear, which was collected by filtration, washed with distilled water and dried at room temperature to afford $0.30 \mathrm{~g}$ of a yellow powder in $99 \%$ yield. ${ }^{1} \mathrm{H}$ NMR (500 MHz, $d^{6}$-DMSO): $\delta 13.24$ (s, 2H, H-16, H-20), 10.46 (s, 2H, H-7, H-10), 7.60 (s, 4H, H-2, H-3, H-5, H-6), 6.47 (d, J = 12.1 Hz, 2H, H-13, H-17), 6.31 (d, $J=12.1 \mathrm{~Hz}, 2 \mathrm{H}, \mathrm{H}-14, \mathrm{H}-18)$.

\subsection{Synthesis of $\left(2 Z, 2^{\prime} Z\right)-4,4^{\prime}-((m e t h y l e n e b i s(4,1-p h e n y l e n e)) b i s(a z a n e d i y l)) b i s(4-o x o b u t-2-$ enoic acid) (4)}

In a $50 \mathrm{~mL}$ two-neck round-bottomed flask, a mixture of $0.20 \mathrm{~g}$ (2.0 equiv.) of maleic anhydride and $5 \mathrm{~mL}$ of acetic acid were heated until reflux. To this mixture, a solution of $0.20 \mathrm{~g}$ (1.0 equiv.) of 4,4'-methylendianiline in $5 \mathrm{~mL}$ of acetic acid was added dropwise. After the addition was completed, the mixture was kept at reflux until a precipitate was formed. The solution was let to cool at RT (room temperature) and $30 \mathrm{~mL}$ of water were added, the product was filtered and washed with an 8:2 water:ethanol mixture and was let to dry, affording $0.24 \mathrm{~g}$ of a white powder in $62 \%$ yield. ${ }^{1} \mathrm{H}$ NMR $\left(500 \mathrm{MHz}, d^{6}-\mathrm{DMSO}\right)$ : $\delta 13.07$ (s, 2H, H-20, H-28), 10.39 (s, 2H, H-7, H-21), 7.54 (d, J = 8.6 Hz, 4H, H-2, H-6, H-13, H-15), 7.17 (d, J = 8.5 Hz, 4H, H-3, H-5, H-12, H-16), 6.46 (d, J = $12.1 \mathrm{~Hz}, 2 \mathrm{H}, \mathrm{H}-17, \mathrm{H}-25)$, 6.29 (d, J = 12.1 Hz, 2H, H-18, H-26), 3.86 (s, 1H, H-10); ${ }^{13} \mathrm{C}$ NMR (126 MHz, d6-DMSO): $\delta$ 166.7 (C-8, C-22), 163.1 (C-19, C-27), 137.0 (C-1, C-14), 136.4 (C-4, C-11), 131.6 (C-17, C-25), 130. 7 (C-18, C-26), 129.0 (C-2, C-6, C-13, C-15), 119.8 (C-3, C-5, C-12, C-16), 40.0 (C-10).

\subsection{Synthesis of (2Z,2'Z)-4,4'-((oxybis(4,1-phenylene))bis(azanediyl))bis(4-oxobut-2-enoic acid)} (6)

In a $50 \mathrm{~mL}$ two-neck round-bottomed flask, a mixture of $0.20 \mathrm{~g}$ (2.0 equiv.) of maleic anhydride and $5 \mathrm{~mL}$ of acetic acid were heated until reflux. To this mixture, a solution of $0.20 \mathrm{~g}$ (1.0 equiv.) of 4,4'-oxydianiline in $5 \mathrm{~mL}$ of acetic acid was added dropwise. After the addition was completed, the mixture was kept at reflux until a precipitate was formed. The solution was let to cool at RT and $30 \mathrm{~mL}$ of water were added, the product was filtered and washed with an 8:2 water:ethanol mixture and was let to dry affording $0.20 \mathrm{~g}$ of a white powder in 50\% yield. ${ }^{1} \mathrm{H}$ NMR (500 MHz, d6-DMSO): $\delta 13.12$ (s, 2H, H-23, H-27), 10.43 (s, 2H, H-14, H-17), 7.62 (d, J= 9.0 Hz, 4H, H-3, H-5, H-10, H-12), 6.98 (d, J= $9.0 \mathrm{~Hz}$, $4 \mathrm{H}, \mathrm{H}-2, \mathrm{H}-6, \mathrm{H}-9, \mathrm{H}-13), 6.47$ (d, J=12.1 Hz, 2H, H-20, H-24), 6.29 (d, J=12.1 Hz, 2H, H21, H-25); ${ }^{13} \mathrm{C}$ NMR (126 MHz, d6-DMSO) $\delta 166.6$ (C-15, C-18), 163.0 (C-22, C-26), 152.9 (C1, C-8), 134.0 (C-4, C-11), 131.8 (C-20, C-24), 130.5 (C-21, C-25), 121.3 (C-3, C-5, C-10, C-12), 118.8 (C-2, C-6, C-9, C-13).

\subsection{Synthesis of (2Z,2'Z)-4,4'-((thiobis(4,1-phenylene))bis(azanediyl))bis(4-oxobut-2-enoic acid)} (8)

In a $50 \mathrm{~mL}$ two-neck round-bottomed flask, a mixture of $0.20 \mathrm{~g}$ (2.0 equiv.) of maleic anhydride and $5 \mathrm{~mL}$ of acetic acid were heated until reflux. To this mixture, a solution of $0.22 \mathrm{~g}$ (1.0 equiv.) of 4,4'-thiodianiline in $5 \mathrm{~mL}$ of acetic acid was added dropwise. After the addition was completed, the mixture was kept at reflux until a precipitate was formed. The solution was let to cool at RT and $30 \mathrm{~mL}$ of water was added; the product was filtered and washed with an 8:2 water:ethanol mixture and was let to dry, affording $0.23 \mathrm{~g}$ of a grey powder in $55 \%$ yield. ${ }^{1} \mathrm{H}$ NMR (500 MHz, $d^{6}$-DMSO): $\delta 12.97(\mathrm{~s}, 2 \mathrm{H}, \mathrm{H}-23, \mathrm{H}-27), 10.47$ (2H, H-14, H-17), 7.63 (d, J = 8.7 Hz, 4H, H-3, H-5, H-10, H-12), 7.29 (d, J = 8.7 Hz, 4H, H2, H-6, H-9, H-13), 6.46 (d, J = 12.0 Hz, 2H, H-20, H-24), 6.30 (d, J = $12.0 \mathrm{~Hz}, 2 \mathrm{H}, \mathrm{H}-21, \mathrm{H}-$ 25); ${ }^{13} \mathrm{C}$ NMR (126 MHz, d6-DMSO): $\delta 166.8$ (C-15, C-18), 163.3 (C-22, C-26), 138.0 (C-4, C11), 131.6 (C-20, C-24), 131.4 (C-3, C-5, C-10, C-12), 130.3 (C-21, C-25), 129.6 (C-1, C-8), 120.4 (C-2, C-6, C-9, C-13). 
4.6. Synthesis of (2Z,2'Z)-4,4'-((sulfonylbis(4,1-phenylene))bis(azanediyl))bis(4-oxobut-2-enoic acid) (10)

In a $50 \mathrm{~mL}$ two-neck round-bottomed flask, a mixture of $0.20 \mathrm{~g}$ (2.0 equiv.) of maleic anhydride and $5 \mathrm{~mL}$ of acetic acid were heated until reflux. To this mixture, a solution of $0.25 \mathrm{~g}$ (1.0 equiv.) of 4,4'-sulfonyldianiline in $5 \mathrm{~mL}$ of acetic acid was added dropwise. After the addition was completed, the mixture was kept at reflux until a precipitate was formed. The solution was let to cool at RT and $30 \mathrm{~mL}$ of water was added; the product was filtered and washed with an 8:2 water:ethanol mixture and was let to dry, affording $0.17 \mathrm{~g}$ of a white powder in $39 \%$ yield. ${ }^{1} \mathrm{H}$ NMR (500 MHz, $\left.d^{6}-\mathrm{DMSO}\right): \delta 12.86$ (s, 2H, H22, H-30), 10.68 (s, 2H, H-9, H-23), 7.88 (d, J = 9.0 Hz, 4H, H-2, H-6, H-14, H-18), 7.82 (d, J $=9.0 \mathrm{~Hz}, 4 \mathrm{H}, \mathrm{H}-3, \mathrm{H}-5, \mathrm{H}-15, \mathrm{H}-17), 6.47$ (d, J = 12.0 Hz, 2H, H-19, H-27), 6.31 (d, J = 12.0 $\mathrm{Hz}, 2 \mathrm{H}, \mathrm{H}-20, \mathrm{H}-28) ;{ }^{13} \mathrm{C}$ NMR (126 MHz, d6-DMSO): $\delta 166.8$ (C-21, C-29), 163.8 (C-10, C24), 143.1 (C-19, C-27), 135.6 (C-4, C-16), 131.6 (C-20, C-28), 130.2 (C-1, C-13), 128.4 (C-2, C-6, C-14, C-18), 119.4 (C-3, C-5, C-15, C-17).

\subsection{Synthesis of $\left(2 Z, 2^{\prime} Z\right)-4,4^{\prime}-((9,10-$ dioxo-9,10-dihydroanthracene-2,6-} diyl)bis(azanediyl))bis(4-oxobut-2-enoic acid) (12)

In a $50 \mathrm{~mL}$ two-neck round-bottomed flask, a mixture of $0.20 \mathrm{~g}$ ( 2.0 equiv.) of maleic anhydride and $5 \mathrm{~mL}$ of acetic acid were heated until reflux. To this mixture, a solution of $0.24 \mathrm{~g}$ (1.0 equiv.) of 2,6-diaminoanthracene-9,10-dione in $5 \mathrm{~mL}$ of acetic acid was added dropwise. After the addition was completed, the mixture was kept at reflux until a precipitate was formed. The solution was let to cool at RT and $30 \mathrm{~mL}$ of water was added; the product was filtered and washed with an 8:2 water:ethanol mixture and was let to dry, affording $0.01 \mathrm{~g}$ of a red powder in $22 \%$ yield. ${ }^{1} \mathrm{H}$ NMR (500 MHz, d6-DMSO): $\delta 12.88$ (s, 2H, H-26, H-30), 10.93 (s, 2H, H-17, H-20), 8.47 (d, J = 2.4 Hz, 2H, H-10, H-11) 8.17 (d, J = $8.5 \mathrm{~Hz}, 2 \mathrm{H}, \mathrm{H}-7, \mathrm{H}-14), 8.07$ (dd, $J=8.6,2.3 \mathrm{~Hz}, 2 \mathrm{H}, \mathrm{H}-8, \mathrm{H}-13), 6.53$ (d, J=12.0 Hz, 2H, H23, H-27), 6.37 (d, J = 11.9 Hz, 2H, H-24, H-28); ${ }^{13} \mathrm{C} \mathrm{NMR} \mathrm{(126} \mathrm{MHz,} \mathrm{d6-DMSO):} \delta 181.2$ (C1, C-4), 166.9 (C-18, C-21), 164.0 (C-25, C-29), 144.3 (C-9, C-12), 134.3 (C-23, C-27), 131.5 (C24, C-28), 130.5 (C-2, C-5), 128.5 (C-3, C-6), 128.3 (C-7, C-14), 123.7 (C-8, C-13), 116.1 (C-10, C-11).

4.8. Synthesis of (2Z,2'Z)-4,4'-((disulfanediylbis(4,1-phenylene))bis(azanediyl))bis(4-oxobut-2enoic acid) (14)

In a $50 \mathrm{~mL}$ two-neck round-bottomed flask, a mixture of $0.20 \mathrm{~g}$ (2.0 equiv.) of maleic anhydride and $5 \mathrm{~mL}$ of acetic acid were heated until reflux. To this mixture, a solution of $0.25 \mathrm{~g}$ (1.0 equiv.) of 4,4'-disulfanediyldianiline in $5 \mathrm{~mL}$ of acetic acid was added dropwise. After the addition was completed, the mixture was kept at reflux until a precipitate was formed. The solution was let to cool at RT and $30 \mathrm{~mL}$ of water was added; the product was filtered and washed with an 8:2 water:ethanol mixture and was let to dry affording $0.15 \mathrm{~g}$ of a grey powder in 34\% yield. ${ }^{1} \mathrm{H}$ NMR (500 MHz, d6-DMSO): $\delta 12.94$ (s, 2H, H-24, H-28), 10.48 (s, 2H, H-15, H-18), 7.64 (d, J = 8.8 Hz, 4H, H-3, H-5, H-11, H-13), 7.47 (d, J = $8.9 \mathrm{~Hz}, 4 \mathrm{H}, \mathrm{H}-2, \mathrm{H}-6, \mathrm{H}-10, \mathrm{H}-14), 6.46$ (d, J = 12.1 Hz, 2H, H-21, H-25), 6.30 (d, J = 12.0 Hz, 2H, H-22, H-26); ${ }^{13} \mathrm{C}$ NMR (126 MHz, d6-DMSO): $\delta 166.8$ (C-16, C-19), 163.3 (C-23, C-27), 138.7 (C-4, C-12), 131.6 (C-21, C-25), 130.3 (C-22, C-26), 129.8 (C-2, C-6, C-10, C-14), 120.2 (C-3, C-5, C-11, C-13).

\subsection{Synthesis of $\left(2 Z, 2^{\prime} Z\right)-4,4^{\prime}-\left(\left(\left(\left[1,1^{\prime}-\right.\right.\right.\right.$ biphenyl $]-4,4^{\prime}$-diylbis(oxy))bis $(4,1-$} phenylene))bis(azanediyl))bis(4-oxobut-2-enoic acid) (16)

In a $50 \mathrm{~mL}$ two-neck round-bottomed flask, a mixture of $0.20 \mathrm{~g}$ (2.0 equiv.) of maleic anhydride and $5 \mathrm{~mL}$ of acetic acid were heated until reflux. To this mixture, a solution of $0.37 \mathrm{~g}$ (1.0 equiv.) of 4,4'-([1,1'-biphenyl]-4,4'-diylbis(oxy))dianiline in $5 \mathrm{~mL}$ of acetic acid was added dropwise. After the addition was completed, the mixture was kept at reflux until a precipitate was formed. The solution was let to cool at RT and $30 \mathrm{~mL}$ of water was 
added; the product was filtered and washed with an 8:2 water:ethanol mixture and was let to dry, affording $0.09 \mathrm{~g}$ of a white powder in $16 \%$ yield. ${ }^{1} \mathrm{H}$ NMR (500 MHz, d $\left.d^{6}-\mathrm{DMSO}\right)$ : ठ 13.13 (s, 2H, H-26, H-41), 10.46 (s, 2H, H-14, H-35), 7.66 (d, J = 9.0 Hz, 4H, H-10, H-12, H18, H-22), 7.63 (d, J = 8.7 Hz, 4H, H-3, H-5, H-30, H-32), 7.06 (d, J= 7.3 Hz, 4H, H-9, H-13, H-19, H-21), 7.05 (d, J = 6.9 Hz, 4H, H-2, H-6, H-29, H-33), 6.48 (d, J = $12.1 \mathrm{~Hz}, 2 \mathrm{H}, \mathrm{H}-23$, $\mathrm{H}-38), 6.31$ (d, J = 12.0 Hz, 2H, H-24, H-39).; ${ }^{13} \mathrm{C}$ NMR (126 MHz, d6-DMSO) ठ 166.7 (C-15, C-36), 163.1 (C-25, C-40), 156.6 (C-8, C-20), 152.2 (C-1, C-28), 134.4 (C-4, C-31, C-11, C-17), 131.8 (C-23, C-38), 130.4 (C-24, C-39), 128.0 (C-10, C-12, C-18, C-22), 121.3 (C-3, C-5, C-30, C-32), 119.5 (C-9, C-13, C-19, C-21), 118.4 (C-2, C-6, C-29, C-33).

Author Contributions: All authors contributed equally to this work. All authors have read and agreed to the published version of the manuscript.

Funding: A.I.-J. acknowledges “Proyecto Apoyado por el Fondo Sectorial de Investigación para la Educación CONACyT-SEP CB-2017-2018 (A1-S-32582)” for financial support. E.G.-Z. thanks CBIUAM-I for financial support.

Institutional Review Board Statement: Not applicable.

Informed Consent Statement: Not applicable.

Acknowledgments: All authors thank Mónica Rincón-Guevara (CBS-UAM-I) for supporting the spectroscopic characterization.

Conflicts of Interest: The authors declare no conflict of interest. The funders had no role in the design of the study; in the collection, analyses, or interpretation of data; in the writing of the manuscript, or in the decision to publish the results.

\section{References}

1. Tabăcăru, A.; Pettinari, C.; Galli, S. Coordination polymers and metal-organic frameworks built up with poly(tetrazolate) ligands. Coord. Chem. Rev. 2018, 372, 1-30.

2. $\quad$ Lustig, W.P.; Mukherjee, S.; Rudd, N.D.; Desai, A.V.; Li, J.; Ghosh, S.K. Metal-organic frameworks: Functional luminescent and photonic materials for sensing applications. Chem. Soc. Rev. 2017, 46, 3242-3285.

3. Lytvynenko, A.S.; Kolotilov, S.V. Electrochemically active coordination polymers: A review. Theor. Exp. Chem. 2016, 52, 197211.

4. Liu, J.; Chen, L.; Cui, H.; Zhang, J.; Zhang, L.; Su, C.-Y. Applications of metal-organic frameworks in heterogeneous supramolecular catalysis. Chem. Soc. Rev. 2014, 43, 6011-6061.

5. Li, J.-R.; Kuppler, R.J.; Zhou, H.C. Selective gas adsorption and separation in metal-organic frameworks. Chem. Soc. Rev. 2009, 38, 1477-1504.

6. Giménez-Marquéz, M.; Hidalgo, T.; Serre, C.; Horcajada, P. Nanostructured metal-organic frameworks and their bio-related applications. Coord. Chem. Rev. 2016, 307, 342-360.

7. Robin, A.Y.; Fromm, K.M. Coordination polymer networks with O- and N-donors: What they are, why and how they are made. Coord. Chem. Rev. 2006, 250, 2127-2157.

8. Kitagawa, S.; Kitaura, R.; Noro, S. Functional porous coordination polymers. Angew. Chem. Int. Ed. 2004, 43, $2334-2375$.

9. Batten, S.R.; Neville, S.M.; Turner, D.R. Coordination Polymers: Design Analysis and Application, 6th ed.; Royal Society of Chemistry: Cambridge, UK, 2009; pp. 172-173.

10. Huang, Z.; Song, H.-B.; Du, M.; Chen, S.-T.; Bu, X.-H. Coordination Polymers Assembled from Angular Dipyridyl Ligands and $\mathrm{Cu}^{\mathrm{II}}, \mathrm{Cd}^{\mathrm{II}}$, Co $\mathrm{Co}^{\mathrm{II}}$ Salts: Crystal Structures and Properties. Inorg. Chem. 2004, 43, 931-944.

11. Niu, L.-E.; Yang, Y.; Qi, X.-H.; Liang, X. Synthesis and spectroscopic studies of Lanthanide(III) complexes with (Z)-4-oxo-4(phenylamino) but-2-enoic acid. Synth. React. Inorg. Met. Org. Chem. 2009, 39, 1-5.

12. Li, M.; Liang, Y.-D.; Wu, Y.-X.; Li, K.-S. Synergistic effect of complexes of ethylenediamine double maleamic acid radical and lanthanum (III) with pentaerythritol on the thermal stability of poly(vinyl chloride). Polym. Degrad. Stabil. 2017, 140, 176-193.

13. Lazarou, K.N.; Psycharis, V.; Perlepes, S.P.; Raptopoulou, C.P. Complexes derived from the copper(II) perchlorate/maleamic acid/2,20-bipyridine and copper(II) perchlorate/maleic acid/2,20-bipyridine reaction systems: Synthetic, reactivity, structural and spectroscopic studies. Polyhedron 2009, 28, 1085-1096.

14. Lazarou, K.N.; Boudalis, A.K.; Perlepes, S.P.; Terzis, A.; Raptopoulou, C.P. Maleamate(-1) and maleate(-2) copper(II)-2,2'-bipyridine complexes: Synthesis, reactivity and structural and physical studies Eur. J. Inorg. Chem. 2009, 4554-4563.

15. Li, B.; Shen, Y.; Wu, H.; Wu, X.; Yuan, L.; Ji, Q. Design, synthesis and biological evaluation of novel 3,4-dihydro- 2(1H)-quinolinone derivatives as potential chitin synthase inhibitors and antifungal agents. Eur. J. Med. Chem. 2020, 195, 112278.

16. Sun, Y.; Zhang, G. Photoinduced decarboxylative amino-fluoroalkylation of maleic anhydride. Chem. Eur. J. 2020, $26,419-422$. 
17. Badru, R.; Singh, B. Triethylamine-Catalyzed Synthesis of Oxazepine from Maleamic Acids. J. Heterocycl. Chem. 2015, 52, 635640.

18. Guevara, J.A.; Trujillo, J.G.; Quintana, D.; Jiménez, H.A.; Arellano, M.G.; Bahena, J.R.; Tamay, F.; Ciprés, F.J. Acetylcholinesterase inhibition by products generated in situ from the transformation of N-arylisomaleimides. Med. Chem. Res. 2018, 27, 9891003.

19. Long, Y.L.; Jiang, W.; Wu, X.; Wang, Q. Grafted copolymerization of N-phenylmaleimide and styrene in porous polyvinyl chloride particles suspended in aqueous solution. Des. Monomers Polym. 2019, 22, 66-78.

20. Kumar, P.P.; Devi, B.R.; Dubey, P.K.; Mohiuddin, S.M.G. PEG-600 mediated simple, efficient and eco-friendly synthesis of Nsubstituted imides and chemoselective $C=C$ reduction. Green Chem. Lett. Rev. 2011, 4, 341-348.

21. Chen, Y.; Tssao, K.; De Francesco, E.; Keillor, J.W. Ring substituent effects on the thiol addition and hydrolysis reactions of Narylmaleimides. J. Org. Chem. 2015, 80, 12182-12192.

22. Kiselev, V.D.; Kashaeva, H.A.; Potapova, L.N.; Kornilov, D.A.; Latypova, L.I.; Konovalov, A.I. Hydrophobic acceleration in the Diels-Alder reaction of 9-hydroxymethylanthracene with N-phenylmaleimide. Russ. Chem. Bull. Int. Ed. 2016, 65, $2202-2205$. 